OPEN ACCESS

Edited by:

Sergio Roa

Center for Applied Medical Research (CIMA), Spain

Reviewed by: Germain Gillet

Université Claude Bernard Lyon 1, France

Almudena Porras,

Complutense University of Madrid, Spain

*Correspondence:

Álvaro J. Obaya

ajobaya@uniovi.es

Specialty section:

This article was submitted to

Molecular and Cellular Oncology,

a section of the journal

Frontiers in Oncology

Received: 10 May 2019 Accepted: 06 August 2019

Published: 22 August 2019

Citation:

Fontanil T, Mohamedi Y, Cobo T, Cal S and Obaya ÁJ (2019) Novel

Associations Within the Tumor Microenvironment: Fibulins Meet ADAMTSs. Front. Oncol. 9:796. doi: 10.3389/fonc.2019.00796

\section{Novel Associations Within the Tumor Microenvironment: Fibulins Meet ADAMTSs}

\author{
Tania Fontanil ${ }^{1,2,3}$, Yamina Mohamedi $^{1,3}$, Teresa Cobo $^{4}$, Santiago $\mathrm{Ca}{ }^{1,3}$ and \\ Álvaro J. Obaya ${ }^{3,5 *}$ \\ 'Departamento de Bioquímica y Biología Molecular, Universidad de Oviedo, Oviedo, Spain, ${ }^{2}$ Departamento de Investigación, \\ Instituto Órdoñez, Oviedo, Spain, ${ }^{3}$ Instituto Universitario de Oncología, IUOPA, Universidad de Oviedo, Oviedo, Spain, \\ ${ }^{4}$ Departamento de Cirugía y Especialidades Médico-Quirúrgicas, Instituto Asturiano de Odontología, Universidad de Oviedo, \\ Oviedo, Spain, ${ }^{5}$ Departamento de Biología Funcional, Área de Fisiología, Universidad de Oviedo, Oviedo, Spain
}

The maintenance of tissue homeostasis in any organism is a very complex and delicate process in which numerous factors intervene. Cellular homeostasis not only depends on intrinsic factors but also relies on external factors that compose the microenvironment or cellular niche. Thus, extracellular matrix (ECM) components play a very important role in maintaining cell survival and behavior, and alterations in the ECM composition can lead to different pathologies. Fibulins and ADAMTS metalloproteases play crucial roles in the upkeep and function of the ECM in different tissues. In fact, members of both of these families of secreted multidomain proteins can interact with numerous other ECM components and thus shape or regulate the molecular environment. Individual members of both families have been implicated in tumor-related processes by exhibiting either pro- or antitumor properties. Recent studies have shown both an important relation among members of both families and their participation in several pathologies, including cardiogenesis or cancer. In this review, we summarize the associations among fibulins and ADAMTSs and the effects elicited by those interactions on cellular behavior.

Keywords: fibulin, ADAMTS, cancer, microenvironment, extracelular matrix

\section{CELLULAR MICROENVIRONMENT}

The extracellular matrix (ECM) is a complex three-dimensional network of proteins and carbohydrates that serves as a support for cells and tissues (1). The cellular function of the ECM makes it a dynamic and versatile compartment through the modulation of the production, degradation, and remodeling of all its components, thus participating in organ development, function, and repairment (2).

The ECM is mainly composed of highly modified proteins, which contributes to its specialization in each organ. In general, the main components of the ECM are macromolecules and fibrous proteins as well as a wide variety of enzymes, including proteases, which are involved in processes such as the maturation, assembly, and renewal of ECM components. Thus, the ECM is responsible for maintaining the homeostasis of each individual tissue and, therefore, of the whole organism. In addition, interactions among ECM components help to mold the cellular microenvironment depending on the functional requirements of the organism (3). In particular, the participation of proteases in ECM processes implies irreversible modifications of their substrates, and proteases are involved in a multitude of crucial processes, such as bone remodeling (4), 
cardiogenesis (5), or even neural development (6). Notably, proteolytic activity can also be modified by a myriad of cofactors, inhibitors, and other modulatory factors. These elements, with the complete substrate repertoire of a particular protease, constitute proteolytic systems (7). Thus, importantly, substrate identification is one of the most important challenges in characterizing a proteolytic enzyme under both normal conditions and pathological alterations. In this context, instances of non-specificity, in which the same protease can process multiple substrates, or redundancy, in which some proteins are targets of more than one protease, can be found. Both situations show that proteolytic systems contain more information than the simple sum of their individual components; thus, each physiological or pathological context can be understood only as the result of the interaction of all ECM components.

In this review, we summarize the increasing number of investigations describing the associations between two families of ECM proteins: ADAMTS metalloproteases and fibulins. Different members of both families have been shown to interact and, through their interaction, modulate biological processes such as organogenesis and cancer development.

\section{FIBULINS}

Fibulins are a main component of elastic fibers in the ECM that endow tissues with the ability to contract after stretching, a property called elastogenesis (8). Elastic fibers are basically composed of two different components: an amorphous elastin core, which is immersed within a fibrillar scaffold composed of microfibrils. In addition, the association of the elastin core with the microfibrils requires the participation of many proteins, among which are associated glycoproteins, latent TGF- $\beta$ binding proteins, interfacial proteins, lysyl oxidases, and members of the fibulin family (9). The fibulin family comprises seven extracellular glycoproteins, all of which include a fibulin-type globular domain, which is also known as domain III, at the carboxy (C)-terminus (10). This common domain is preceded by domain II, which includes a variable number of epithelial growth factor (EGF)-like modules, some of which contain a calcium-binding consensus sequence; these domains are known as calcium-binding EGF (cbEGF)-like domains. Finally, domain I is located at the amino-terminus and shows the highest structural variability among the family members (11) (Figure 1).

By their architecture, mammalian fibulins can be classified into two subgroups (12). Subgroup I comprises fibulin-1 and fibulin-2 (100 and $200 \mathrm{kDa}$, respectively) and is characterized by the three anaphylatoxin modules in domain I. In addition, fibulin-2 differs from fibulin-1 because it contains two regions in domain I with additional differences: one rich in cysteines (the cys-rich domain) and the other lacking this amino acid (the cys-free domain).

Subgroup II is formed by the rest of the members but also shows important differences. Fibulin-3, fibulin-4, and fibulin-5 are also known as short fibulins since their molecular weights range from 50 to $60 \mathrm{kDa}$. Fibulin- 6 is the largest fibulin in the family because it possesses an important number of different motifs in domain I: a von Willebrand factor motif, 44 immunoglobulin C2 modules, and 6 thrombospondin type I (TSP-1) motifs. Fibulin-7 is characterized by a "sushi" motif in domain I, which is involved in protein-protein interactions (13) (Figure 1).

\section{THE ADAMTS FAMILY}

The ADAMTS family, whose acronym comes from "A Disintegrin And Metalloprotease with ThromboSpondin

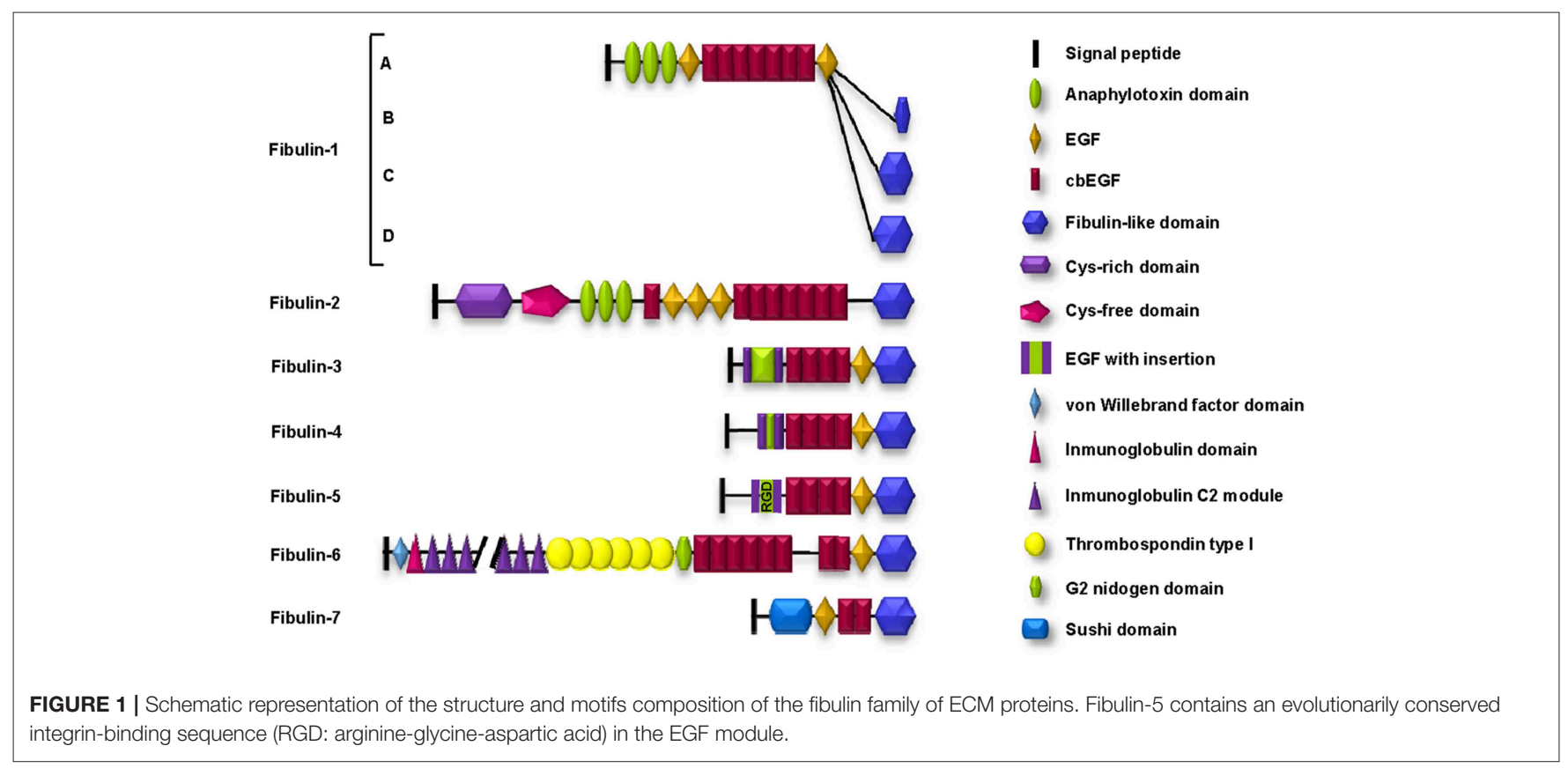


domains," consists of ECM-secreted enzymes whose main characteristic is a set of different adhesion motifs together with a proteolytic motif within the same protein unit. ADAMTSs belong to the metzincin superfamily; thus, their protease domains share great similarity with those of the other members of this family. In this superfamily of proteases, the proteolytic domain is characterized by a zinc atom at the active center and a conserved methionine residue at the carboxy-terminus that forms the characteristic "methionine spin" (14).

The putative adhesion functions of adamalysins arise from the disintegrin domain and cys-rich domain. In addition, ADAMTSs also contain a variable number of TSP-1 motifs that are involved in interactions with multiple factors of the ECM. Functionally, adamalysins are involved in fundamental processes such as cellular interactions or intracellular signaling $(15,16)$.

A total of 19 ADAMTSs have been identified in mammals (17) (Figure 2). The structural organization of ADAMTSs consists of a metalloprotease domain characterized by an aspartic residue at the end of the consensus $\mathrm{Zn}^{2+}$-chelating motif HExxHxxGxxHD, a disintegrin domain, a spacer domain, a mucin-like domain and several TSP-1 domains. One TSP-1 domain is located between the disintegrin domain and the cys-rich domain, and the rest reside in variable numbers at the carboxy-terminus of the protein. Other motifs, such as a GON-1 motif, are found in some members of the family, such as ADAMTS-9 and ADAMTS-20, and a cubillin (cub) motif is found only in ADAMTS-13 (18-20). The carboxyterminal region of ADAMTSs is extraordinarily variable and gives these enzymes many of their unique properties. All domains in this region are mainly involved in adhesion and anchorage functions and profoundly affect the manner in which the protein interacts with its substrates as well as with other components of the ECM or the cellular surface. Notably, the term orphan ADAMTS, employed by Porter et al. (17) in 2005 to refer to
ADAMTSs with no known substrate or function, can still be used today to refer to some members of the family.

\section{FIBULIN-1 AND ITS ASSOCIATIONS WITH ADAMTSS}

The gene coding for fibulin-1, FBLN-1, also known as BM-90, was identified in 1989 as the first member of the fibulin family (21). FBLN-1 gene, located at human chromosome position 22 p13, contains 20 exons and undergoes splicing to produce four mRNA variants. Thus, four different forms of fibulin-1 can be identified: fibulin-1A, fibulin-1B, fibulin-1C, and fibulin-1D. They differ in both the length and amino acid sequence of their terminal fibulin-like domain $(22,23)$.

In relation to tumor processes, fibulin-1 shows a dual function, since both oncogenic and tumor-suppressive properties have been described for this protein. For example, low expression of fibulin-1 is associated with poor prognosis in gastric, colon, and lung cancers (24-26). In other studies, epigenetic downregulation by hypermethylation has been described in various cancers, such as gastric, renal, colorectal, hepatocellular, and bladder cancers (27-31). However, fibulin-1 expression in the stroma is associated with increased malignancy in ovarian and breast cancers (32). Thus, the ambiguous function associated with fibulin-1 in tumor-related processes has several possible explanations: posttranslational modifications, such as the proteolytic processes affecting fibulin-1 in mammary tumors; alternative splicing, which generates the different forms of fibulin-1; interaction of fibulin-1 with different components of the ECM; or even the areas where fibulin-1 is expressed [stromal vs. tumor cells; (22, 33-35)].

ADAMTS-1 is an ECM component that can interact with fibulin-1 (36). ADAMTS-1 was the first identified member of

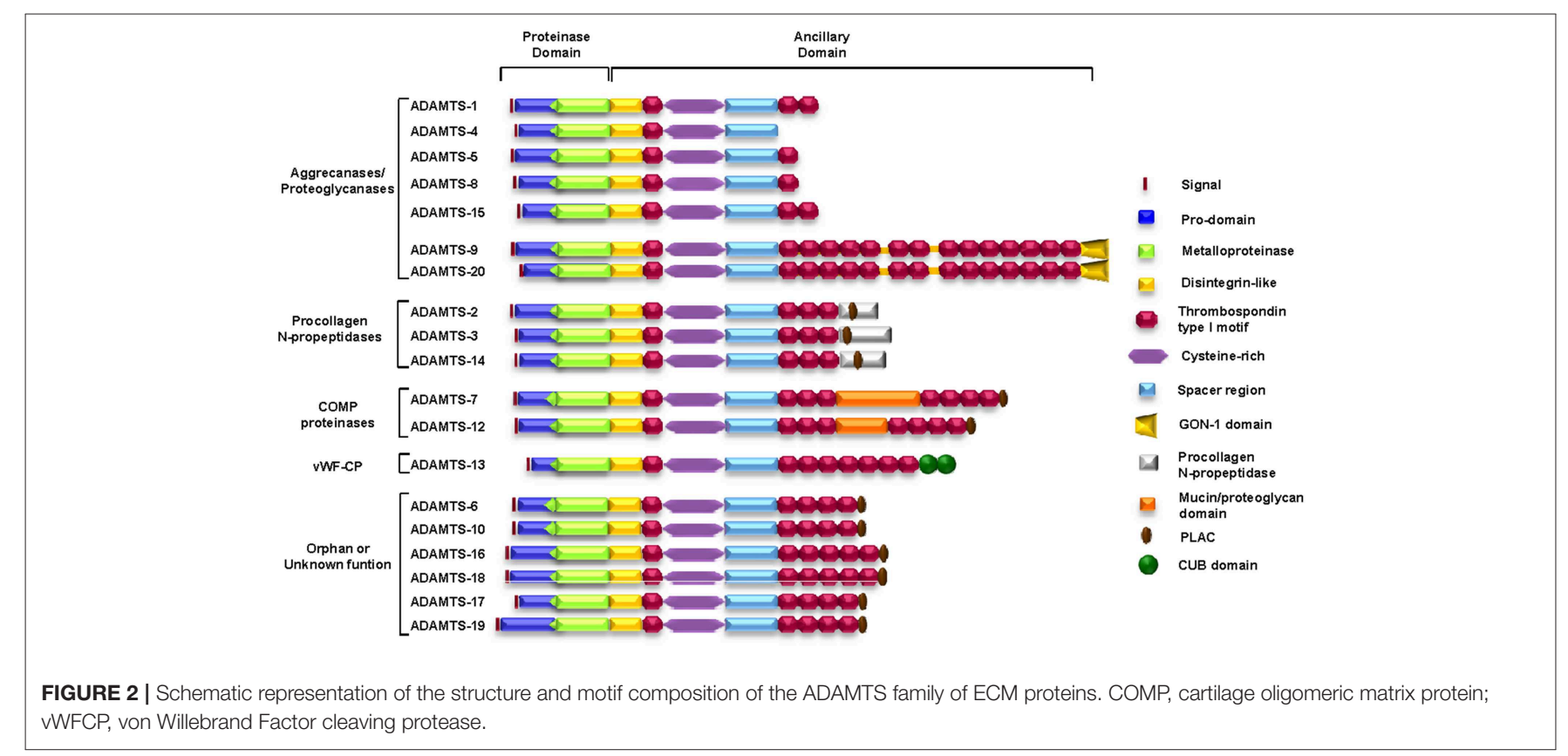


the ADAMTS family, and its activation is mediated by a furinlike enzyme, leading to the elimination of the pro domain. Once activated, the enzyme may undergo a secondary processing event that separates the catalytic subunit from the thrombospondin repeats (37).

Like fibulin-1, ADAMTS-1 has also been implicated to participate in tumor processes with a dual function. Initially, antiangiogenic properties were associated with ADAMTS-1 through the inhibition of vascularization induced by basic fibroblast growth factor (bFGF) in corneal pocket assays. In addition, ADAMTS-1 blocked the angiogenesis process promoted by vascular endothelial growth factor (VEGF) in chorioallantoic membrane assays $(38,39)$. In addition, ADAMTS-1 can block tumor growth in vivo by sequestering VEGF165 through its carboxy-terminal region, which contains the final two TSP-1 motifs (40). In stark contrast, different studies have also demonstrated that this enzyme may elicit tumorprotective properties. For instance, ADAMTS-1 can promote tumor growth and progression in both breast and ovarian cancers (41-43) and can induce pathological angiogenesis through versican degradation (44). Additionally, this metalloprotease has been described to participate in a stromal reaction directed toward recruiting fibroblasts to participate in tumor growth (45).

These apparently contradictory results can be explained in different ways. The pro- or antitumor effects have been suggested to be conditioned by the different fragments derived from autolysis of the enzyme (38). Other factors that may also affect the balance between the pro- or antitumor properties of ADAMTSs are the interaction with other components of the ECM or even the formation of bioactive cleavage products (46). Fibulin-1 binds to ADAMTS-1 through the last three replicates of the fibulin-like domain, similar to the TSP-1 motifs found in the carboxy-terminus of ADAMTS-1 (Figure 3); this interaction was confirmed by various in vitro and in vivo biochemical assays (36). The presence of fibulin-1 increases the catalytic activity of ADAMTS-1 to degrade aggrecan, resulting in the generation of two specific proteolytic fragments of 250 and $65 \mathrm{KDa}$. In fact, the level of fibulin-1 and an increase in aggrecan degradation by ADAMTS-1 are positively correlated. Fibulin-1 acts through altering the conformation of the aggrecan molecule and thus facilitating its proteolytic processing by the ADAMTS-1 metalloprotease (36). Versikine is a recently described bioactive product derived from the specific proteolytic degradation of versican (Figure 3) (47). Versikine participates in cellular functions in cancer development by regulating processes such as the apoptosis and migration of immune cells (48-50).

Versican proteolysis favored by the action of fibulin-1 as a cofactor of ADAMTS family members also occurs in interdigital web regression in mammals. Versican, a widely distributed proteoglycan in embryonic ECM, has been shown to interact

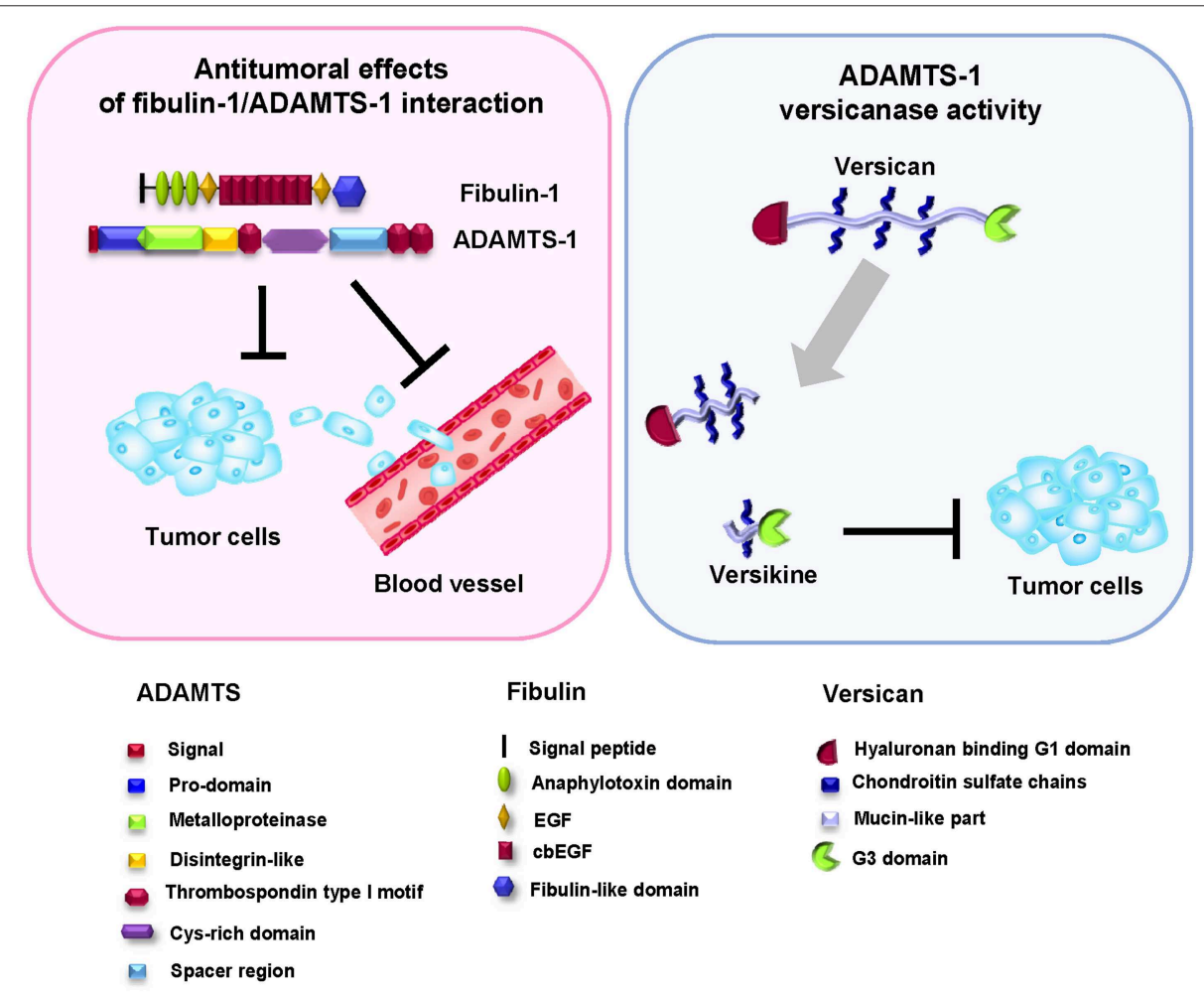

FIGURE 3 | Antitumor effect of the fibulin-1/ADAMTS-1 interaction. Left, the fibulin-1/ADAMTS-1 interaction and its effects on tumor development. Right: ADAMTS-1 shows versicanase activity, which is potentiated by the presence of fibulin-1. Versikine, a byproduct of versican degradation, also shows antitumor properties. Bottom, protein motifs of ADAMTS, fibulin, and versican. 
with fibulin-1 through its carboxy-terminal G3 domain (51). Similar to its relationship with ADAMTS-1, versican is also a known substrate of ADAMTS-5 (52). Interdigital web regression requires the removal of not only interdigital cells, which occurs by apoptosis, but also the ECM (48). In this sense, and considering all these components of the ECM, ADAMTS-5 is the most active known versicanase among the proteases participating in the interdigital web regression process. In a similar way to that occurring in cardiogenesis or tumor processes, versican degradation by proteolysis is a critical event in web regression. Fibulin-1 regulates this process by enhancing the proteolytic activity of ADAMTS-5 and increasing the generation of a 70 $\mathrm{kDa}$ fragment from versican during interdigital tissue (IDT) regression. Finally, this fragment of versican is located in the population of cells destined to undergo apoptosis. This finding suggests that fibulin- 1 acts as a cofactor for ADAMTS-5 in IDT regression (48).

In a recent study, the interaction between fibulin-1 and ADAMTS-1 was associated with antitumor effects in breast cancer cell lines (35). In fact, over-expression of exogenous ADAMTS-1 and fibulin-1 resulted in a reduction in the invasive and migratory phenotypes of these cell lines. In

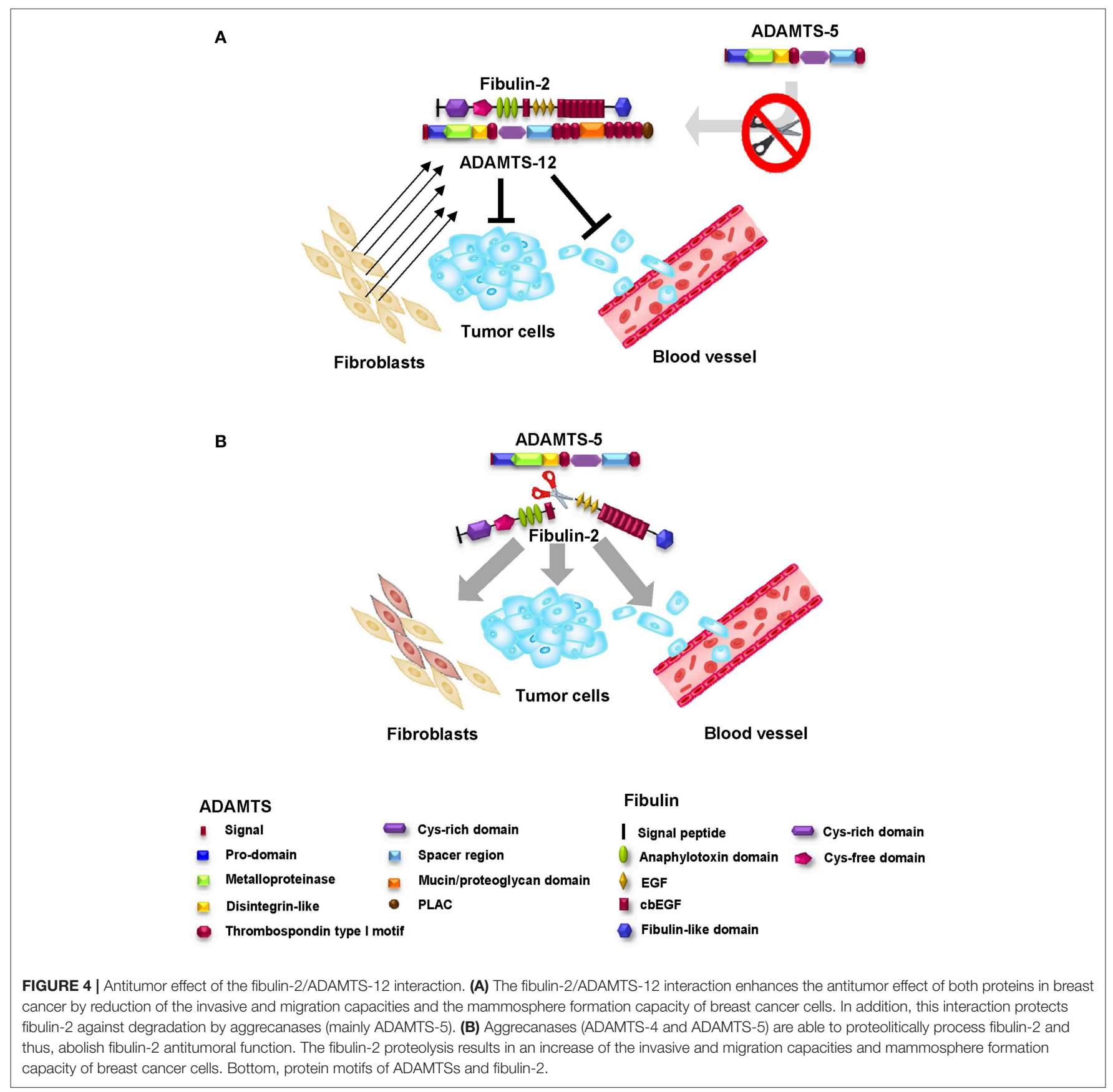


addition, the presence of both proteins also reduced the mammosphere formation capacity in in vitro experiments. In contrast, in the absence of fibulin-1, ADAMTS-1 shows a protumor function, indicating that the fibulin-1/ADAMTS-1 interaction could be considered a good prognostic factor in mammary tumors.

Fibulin-1 and ADAMTS-1 also seem to play crucial roles in other physiological events, such as the corneal formation or cardiogenesis $(53,54)$. By microarray analysis, fibulin1 and ADAMTS-1 expression was detected in primary and immortalized human corneal fibroblasts $(\mathrm{CHN})$, along with other components of the ECM known to interact with both proteins, such as nidogen-1 and aggrecan. The participation of fibulin-1 in cell migration was demonstrated after siRNA transfection of CHN cells, but cell adhesion was not affected (53). However, fibulin-1 participates in cardiogenesis by its high expression at sites of epithelial-mesenchymal transformation (EMT), in the endocardial cushion, in the coronary vessels and in the embryonic myocardium (55). Embryonic expression of fibulin-1 is considered transient in most tissues and disappears during development. However, fibulin-1 regulates versican-dependent events during ventricular morphogenesis (56). Versican is a proteoglycan essential for the formation of the endocardial cushion mesenchyme through EMT. As described for cancer development, ADAMTS- 1 is an important factor in regulating versican activity through versican proteolysis events. Here, versican proteolysis results in the release of a $70 \mathrm{kDa}$ fragment of versican, which is fundamental in the development and differentiation of the endocardial cushion mesenchyme (54). ADAMTS-1 as well as its cofactor fibulin-1, is expressed during early heart development $(55,57)$. The expression of both proteins suggests that fibulin-1 regulates the whole process of morphogenesis by enhancing the proteolytic activity of ADAMTS-1 toward versican and thus promoting the proliferation of cardiac cushion cells $(55,58,59)$.

\section{ASSOCIATIONS OF FIBULIN-2 WITH ADAMTSS}

Fibulin-2 was discovered in 1993 from sequence analysis of cDNA clones obtained from a mouse fibroblast library. The FBLN2 gene is located at the p24-p25 region of human chromosome 3 and in the D-E band of mouse chromosome 6. Fibulin-2 possesses two distinct domains called the cys-rich and cys-free segments within its $\mathrm{N}$-terminal region (60). This wide variety of domains serves to establish interactions with multiple ECM and cell surface components. For example, fibulin-2 can interact with aggrecan, nidogen, fibronectin, and perlecan, etc., as well as with cellular integrins (61-64), thereby contributing to the maintenance of extracellular structures such as basement membranes and elastic fibers (10).

The complexity of the functions of fibulin-2 is also evident in pathological conditions such as cancer $(11,65)$. As with fibulin-1, opposing functions have been described for fibulin-2. For example, protumor effects are associated with fibulin-2 in pancreatic cancer as a consequence of its interaction with type I transmembrane glycoprotein MUC4. This interaction might disrupt the integrity of the basement membrane, and thus promote the metastatic process (66). However, the antitumor properties of fibulin-2 are the most well-known, for example, in nasopharyngeal carcinoma, where fibulin-2 acts as an antiangiogenic factor (67), or in breast cancer, where the reduction in fibulin-2 expression facilitates tumor progression, increasing cellular migratory and invasive properties (68). In addition, the human gene encoding fibulin-2 is epigenetically silenced in acute Bcell lymphoblastic leukemia (69). Collectively, these studies show the importance of fibulin-2 in tumor development. However, the reason for this dual role in cancer is again believed to be due, similar to fibulin-1, to other factors such as posttranslational modifications, interactions with other components of the ECM, fibulin-2 site of expression within the cancerous tissue or even the clinical grade or stage of the tumor $(70,71)$.

Previous studies from our laboratory revealed that fibulin2 is a molecular partner of ADAMTS-12 (72) (Figure 4A). ADAMTS-12 is a secreted metalloprotease associated with important roles in tissue remodeling, inflammation, cell migration, and adhesion (73-75). Regarding tumor-related processes, ADAMTS-12 has also been implicated to have a dual effect, described as a protumor factor as well as an antitumor factor. The participation of ADAMTS-12 with an antitumoral function has been described in colorectal carcinomas with silencing of the ADAMTS12 gene promoter by hypermethylation (76). This function is also suggested by the inhibition of the scattering of Madin-Darby canine kidney (MDCK) cells in the presence of HGF (75). MDCK cells in the presence of HGF experience both, a scattering effect and a tubulogenesis process, that ends in the formation of invasive extensions characteristic of a partial epithelial-mesenchymal transition (EMT) (77). Thus, abolishing this event by the presence of ADAMTS-12 can be considered as an antitumoral effect of this metalloprotease. Finally, studies with ADAMTS-12-deficient mice also indicate this antitumor phenotype (78). By using a model of induced tumors after malignant keratinocytes transplantation, the absence of ADAMTS-12 in Adamts12-/mice results in an increase in the angiogenic response and in tumor growth when compared with wild type mice. Furthermore, capillary outgrowth from mouse aortic rings cultured on a collagen gel was greater in aortic explants from Adamts12-/mice. The addition of conditioned culture medium obtained from cells that express ADAMTS-12 is able to suppress this capillary outgrowth also suggesting an antitumoral role for this protease (78).

The interaction between fibulin-2 and ADAMTS-12 occurs between the spacer-1 region of ADAMTS-12 and the carboxyterminus of fibulin-2 (72). This interaction enhances the antitumor effect of both proteins in breast cancer, reducing the invasive capacity, migration, and the mammosphere formation capacity of the cells. In this context, where both proteins are exogenously over-expressed, the fibulin-2/ADAMTS-12 complex not only affects the cellular properties of breast cancer cell 
lines but also diminishes the growth of subcutaneous tumors in mice (72).

Another obvious demonstration of the importance of fibulin2 within the tumor microenvironment is derived from the observation that it can be cleaved by the metalloproteases ADAMTS-4 and, especially, ADAMTS-5 (79) (Figure 4B). ADAMTS- 4 and ADAMTS-5 are enzymes closely related to bone pathologies such as osteoarthritis due to their high aggrecanase activity (80, 81). Regarding tumor-related processes, both ADAMTS-4 and ADAMTS-5 are associated with a protumor role. In fact, the presence of both enzymes increases the invasive potential of glioblastoma cancer cells through brevican degradation $(82,83)$, and high levels of both ADAMTSs are related to an increase in the tumorigenic potential of ovarian carcinomas (43). Previous works have revealed that fibulin-2 can be proteolytically processed by MMPs and serine proteases involved in tissue remodeling (84). Our data regarding the cleavage of fibulin- 2 by ADAMTS- 5 strengthen the tumorprotective role of fibulin-2 in breast cancer. In fact, cleaved fibulin-2 is associated with an increase in the invasive phenotype of breast cancer cells; in addition, this processing can modify the nature of the surrounding fibroblasts by conferring protumor properties (79). However, there is a balance between the proand antitumor effects of fibulin-2 that seems to depend mainly on ADAMTS-12, which-as previously mentioned-interacts with fibulin-2, affording protection against cancer. However, in addition, this protective effect is extended by protecting fibulin-2 from degradation by ADAMTS-4 and ADAMTS-5. These effects highlight the importance of both fibulins and ADAMTSs as modifiers of the tumor microenvironment.

\section{FIBULIN-3 AND ADAMTS-5}

Fibulin-3, also known as EFEMP1, is a protein located in the ECM of elastic tissues (12). Fibulin-3 may act as a regulator of cell growth and could influence the development of tumors. In support of these roles, fibulin-3 expression is increased in transformed cell lines compared to that in normal controls (85). In addition, when fibulin-3 mRNA is microinjected into normal human diploid fibroblasts (HDF normal cells), they undergo an increase in the rate of DNA synthesis compared to that in cells that have not been microinjected (65). However, fibulin-3 is absent from normal brain tissue $(12,86)$ and is downregulated in several types of solid tumors $(87,88)$. Surprisingly, fibulin3 is upregulated in gliomas, where it promotes tumor growth and invasion (88). Recent results suggest that fibulin-3 is also upregulated in some highly metastatic tumors, where it correlates with the progression of these tumors toward the invasive phenotype $(89,90)$. Fibulin-3 participation in cancer implies different mechanisms. For example, fibulin-3 overexpression can be associated with a malignant phenotype and poor prognosis of cervical carcinoma. In this case, fibulin-3 promotes EMT by activation of the PI3K-Akt-mTOR signaling pathway (91). In osteosarcoma, fibulin-3 upregulation was positively correlated with poor prognosis and is able to promote cell invasion and metastasis by inducing EMT and activating the $\mathrm{Wnt} / \beta$ catenin signaling pathway (92). Furthermore, fibulin-3 also stimulates migration and invasion of HCT116 cells through mechanisms involving p38 $\alpha$ MAPK activation (93). In other cases, high levels of fibulin-3 are responsible for inhibiting EMT as well as migration, invasion and endothelial permeability

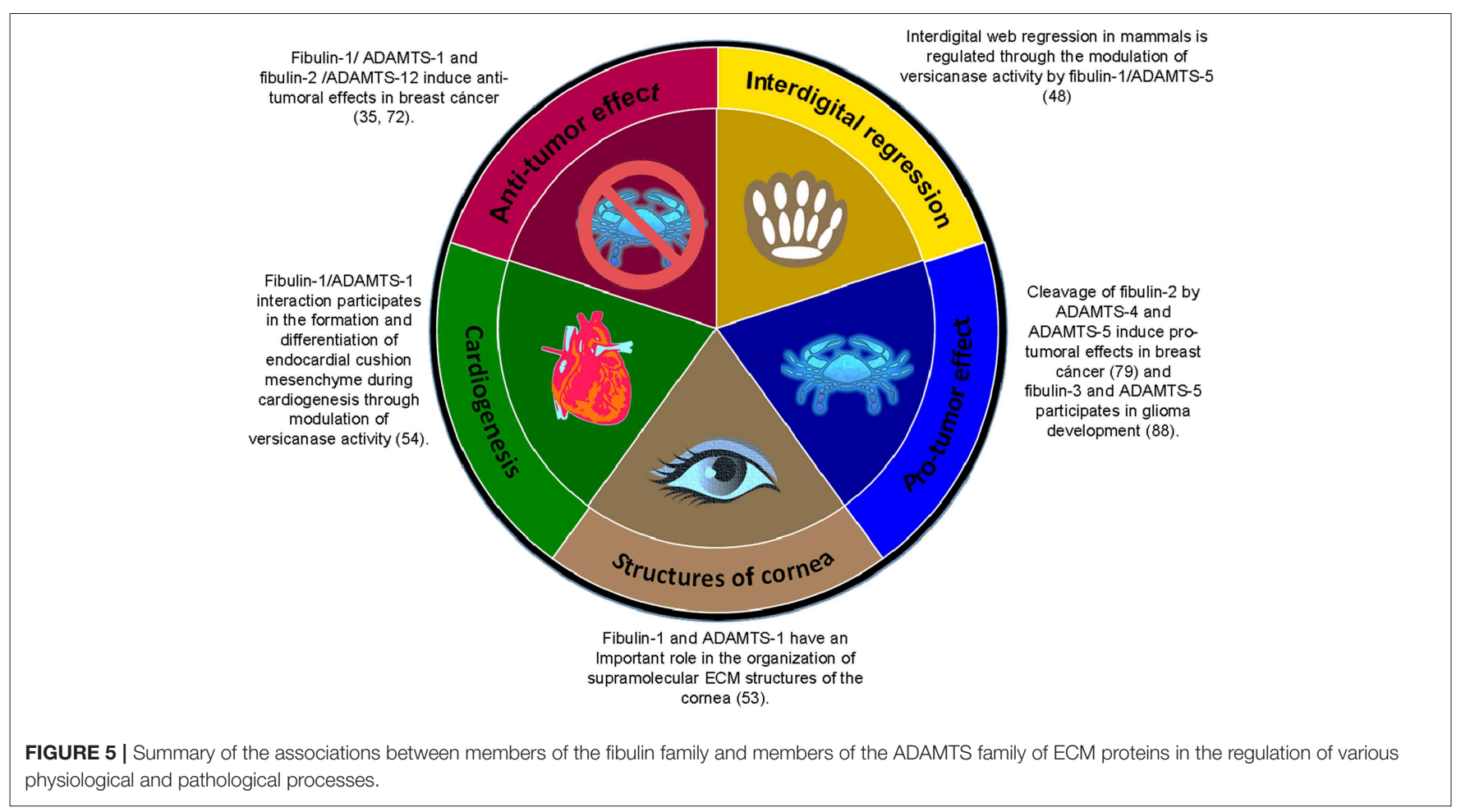


processes in breast cancer microenvironment through the TGF- $\beta$ mediated pathway (94). In lung cancer, fibulin-3 downregulation is required to promote invasion and metastasis by $\mathrm{Wnt} / \beta$-catenin activation and MMP-7 expression (95). In summary, fibulin-3 participation in cancer might depend on the involved pathways, protein-protein interactions and tumor microenvironment.

A proposed mechanism by which fibulin-3 acts in tumorrelated processes is as a signal to promote cell invasion and tumor dispersion. Consistent with this proinvasive role, CNS-1 glioma cell-induced tumors that overexpress fibulin-3 have significantly upregulated levels of mRNAs coding for several matricellular proteases, including MMP-2, MMP-9, and ADAMTS-5, all of which are involved in pericellular ECM degradation, glioma invasion, and intracranial tumor dispersion. In addition, cultured CNS-1 cells overexpressing fibulin-3 exhibited significant positive regulation of the same metalloproteases at the mRNA and protein levels as well as a strong increase in metalloprotease activity, suggesting a direct regulatory effect of fibulin-3 on the expression of metalloproteases such as ADAMTS-5 and the proteolytic activity observed in glioma cells (88).

\section{CONCLUSION}

The ECM that forms any given tissue is a determinant for numerous cellular processes. Certain components can modify this environment, leading to the generation of organs or to the development of pathologies, such as cancer. Thus, the ECM plays an important role in cellular behavior and, as a consequence, can be a force in remodeling the cellular environment and, consequently, cellular properties.

In this review, we focused on the relationship between two families of ECM proteins: fibulins and ADAMTSs (Figure 5). Among the fibulins, fibulin-1 is the best characterized member, with the greatest number of described partners. In fact, this protein seems to play a more relevant role than its related family member fibulin-2, considering the characteristics of fibulin-1deficient mice. Fibulin-1 knockout mice do not survive beyond birth because of bleeding in the muscle, skin and perineural tissues (96) while fibulin-2 deficient mice are viable, fertile and do not exhibit apparent phenotypic defects (97). The function of fibulin-2 seems to be compensated for by an increase in fibulin-1 expression, which, in turn, compensates for the absence of fibulin-2. Fibulin-1 and fibulin-2 can interact with two related members of the ADAMTS family of proteases,

\section{REFERENCES}

1. Bonnans C, Chou J, Werb Z. Remodelling the extracellular matrix in development and disease. Nat Rev Mol Cell Biol. (2014) 15:786-801. doi: $10.1038 / \mathrm{nrm} 3904$

2. Page-McCaw A, Ewald AJ, Werb Z. Matrix metalloproteinases and the regulation of tissue remodelling. Nat Rev Mol Cell Biol. (2007) 8:221-33. doi: $10.1038 / \mathrm{nrm} 2125$

3. Marinkovic M, Block TJ, Rakian R, Li Q, Wang E, Reilly MA, et al. One size does not fit all: developing a cell-specific niche for
ADAMTS-1 and ADAMTS-12, respectively. These interactions can modify the protumor properties exhibited by ADAMTS1 and ADAMTS-12 in breast cancer cells. Consequently, the simultaneous presence of fibulin-1 and ADAMTS-1 or of fibulin-2 and ADAMTS-12 could be considered a good prognostic factor in breast cancer. Moreover, fibulin-1 is a known cofactor of the proteolytic activity of ADAMTS1 toward some of its substrates, such as versican. Fibulin1 is also implicated with ADAMTS-5 in other physiological processes such as interdigital web regression by modulation of its versicanase activity. However, ADAMTS-5 also cooperates with fibulin-3 to promote cell invasion in gliomas through proteolytic degradation of ECM components. Finally, ADAMTS5 and ADAMTS-4 interact with and can degrade fibulin-2. Interestingly, the degradation of fibulin- 2 by either ADAMTS4 or ADAMTS-5 is blocked by the presence of ADAMTS12 and seems to be a target for the protective role of the fibulin-2/ADAMTS-12 interaction in breast cancer (Figure 5). Collectively, these studies demonstrate the importance and increasing number of interactions between members of both of these families of secreted multidomain proteins. The exploration of new associations between fibulins and ADAMTS would help to shed light on not only the maintenance of tissue homeostasis but also the involvement of these proteins in pathological processes.

\section{AUTHOR CONTRIBUTIONS}

All authors listed have made a substantial, direct and intellectual contribution to the work, and approved it for publication.

\section{FUNDING}

This work was supported by the Instituto Asturiano de Odontología (IAO). TF is the recipient of a contract from the Departamento de Investigación Instituto Órdoñez (Oviedo), and YM is the recipient of a fellowship from the IAO and a former recipient of a fellowship from the Fundación para el Fomento en Asturias de la Investigación Científica Aplicada y la Tecnología (FICYT).

\section{ACKNOWLEDGMENTS}

We would like to thank J. A. Vega and J. Cobo for the critical reading of the manuscript.

in vitro study of cell behavior. Matrix Biol. (2016) 52-54:426-41 doi: 10.1016/j.matbio.2016.01.004

4. Alford AI, Kozloff KM, Hankenson KD. Extracellular matrix networks in bone remodeling. Int J Biochem Cell Biol. (2015) 65:20-31. doi: 10.1016/j.biocel.2015.05.008

5. Bowers SL, Baudino TA. Laying the groundwork for growth: cell-cell and cellECM interactions in cardiovascular development. Birth Defects Res C Embryo Today. (2010) 90:1-7. doi: 10.1002/bdrc.20168

6. Ranga A, Girgin M, Meinhardt A, Eberle D, Caiazzo M, Tanaka EM, et al. Neural tube morphogenesis in synthetic $3 \mathrm{D}$ microenvironments. 
Proc Natl Acad Sci USA. (2016) 113:E6831-E9. doi: 10.1073/pnas.16035 29113

7. Lopez-Otin C, Overall CM. Protease degradomics: a new challenge for proteomics. Nat Rev Mol Cell Biol. (2002) 3:509-19. doi: 10.1038/nrm858

8. Wagenseil JE, Mecham RP. New insights into elastic fiber assembly. Birth Defects Res C Embryo Today. (2007) 81:229-40. doi: 10.1002/bdrc.20111

9. Segade F. Molecular evolution of the fibulins: implications on the functionality of the elastic fibulins. Gene. (2010) 464:17-31. doi: 10.1016/j.gene.2010.05.003

10. de Vega S, Iwamoto T, Yamada Y. Fibulins: multiple roles in matrix structures and tissue functions. Cell Mol Life Sci. (2009) 66:1890-902. doi: 10.1007/s00018-009-8632-6

11. Obaya AJ, Rua S, Moncada-Pazos A, Cal S. The dual role of fibulins in tumorigenesis. Cancer Lett. (2012) 325:132-8. doi: 10.1016/j.canlet.2012.06.019

12. Kobayashi N, Kostka G, Garbe JH, Keene DR, Bachinger HP, Hanisch FG, et al. A comparative analysis of the fibulin protein family. Biochemical characterization, binding interactions, and tissue localization. J Biol Chem. (2007) 282:11805-16. doi: 10.1074/jbc.M611029200

13. de Vega S, Iwamoto T, Nakamura T, Hozumi K, McKnight DA, Fisher LW, et al. TM14 is a new member of the fibulin family (fibulin-7) that interacts with extracellular matrix molecules and is active for cell binding. J Biol Chem. (2007) 282:30878-88. doi: 10.1074/jbc.M705847200

14. Gomis-Ruth FX. Structural aspects of the metzincin clan of metalloendopeptidases. Mol Biotechnol. (2003) 24:157-202. doi: $10.1385 / \mathrm{MB}: 24: 2: 157$

15. Paulissen G, Rocks N, Gueders MM, Crahay C, Quesada-Calvo F, Bekaert S, et al. Role of ADAM and ADAMTS metalloproteinases in airway diseases. Respir Res. (2009) 10:127. doi: 10.1186/1465-9921-10-127

16. Kumar S, Rao N, Ge R. Emerging roles of ADAMTSs in angiogenesis and cancer. Cancers. (2012) 4:1252-99. doi: 10.3390/cancers4041252

17. Porter S, Clark IM, Kevorkian L, Edwards DR. The ADAMTS metalloproteinases. Biochem J. (2005) 386(Pt 1):15-27. doi: 10.1042/BJ20 040424

18. Gurses MS, Ural MN, Gulec MA, Akyol O, Akyol S. Pathophysiological function of ADAMTS enzymes on molecular mechanism of Alzheimer's disease. Aging Dis. (2016) 7:479-90. doi: 10.14336/AD.2016.0111

19. Llamazares M, Cal S, Quesada V, Lopez-Otin C. Identification and characterization of ADAMTS-20 defines a novel subfamily of metalloproteinases-disintegrins with multiple thrombospondin-1 repeats and a unique GON domain. J Biol Chem. (2003) 278:13382-9. doi: $10.1074 /$ jbc.M211900200

20. Cal S, Obaya AJ, Llamazares M, Garabaya C, Quesada V, LopezOtin C. Cloning, expression analysis, and structural characterization of seven novel human ADAMTSs, a family of metalloproteinases with disintegrin and thrombospondin-1 domains. Gene. (2002) 283:49-62. doi: 10.1016/s0378-1119(01)00861-7

21. Argraves WS, Dickerson K, Burgess WH, Ruoslahti E. Fibulin, a novel protein that interacts with the fibronectin receptor beta subunit cytoplasmic domain. Cell. (1989) 58:623-9.

22. Argraves WS, Greene LM, Cooley MA, Gallagher WM. Fibulins: physiological and disease perspectives. EMBO Rep. (2003) 4:1127-31. doi: 10.1038/sj.embor.7400033

23. Timpl R, Sasaki T, Kostka G, Chu ML. Fibulins: a versatile family of extracellular matrix proteins. Nat Rev Mol Cell Biol. (2003) 4:479-89. doi: 10.1038/nrm1130

24. Feng L, Yao C, Li P, Feng Y, Wang F, Liu YF, et al. Low expression of fibulin-1 correlates with unfavorable prognosis in gastric cancer. Tumour Biol. (2016) 37:9399-410. doi: 10.1007/s13277-015-4537-1

25. Zhu J, Chen R, Mo L, Tang H, Kuang Y, Fei W, et al. Expression of fibulin-1 predicted good prognosis in patients with colorectal cancer. Am J Transl Res. (2015) 7:339-47.

26. Cui Y, Liu J, Yin HB, Liu YF, Liu JH. Fibulin-1 functions as a prognostic factor in lung adenocarcinoma. Jpn J Clin Oncol. (2015) 45:854-9. doi: 10.1093/jjco/hyv094

27. Xu Z, Chen H, Liu D, Huo J. Fibulin-1 is downregulated through promoter hypermethylation in colorectal cancer: a CONSORT study. Medicine. (2015) 94:e663. doi: 10.1097/MD.0000000000000663
28. Xiao W, Wang J, Li H, Xia D, Yu G, Yao W, et al. Fibulin-1 is epigenetically down-regulated and related with bladder cancer recurrence. BMC Cancer. (2014) 14:677. doi: 10.1186/1471-2407-14-677

29. Cheng YY, Jin H, Liu X, Siu JM, Wong YP, Ng EK, et al. Fibulin 1 is downregulated through promoter hypermethylation in gastric cancer. $\mathrm{Br} \mathrm{J}$ Cancer. (2008) 99:2083-7. doi: 10.1038/sj.bjc.6604760

30. Kanda M, Nomoto S, Okamura Y, Hayashi M, Hishida M, Fujii T, et al. Promoter hypermethylation of fibulin 1 gene is associated with tumor progression in hepatocellular carcinoma. Mol Carcinog. (2011) 50:571-9. doi: $10.1002 / \mathrm{mc} .20735$

31. Xiao W, Wang J, Li H, Guan W, Xia D, Yu G, et al. Fibulin-1 is down-regulated through promoter hypermethylation and suppresses renal cell carcinoma progression. J Urol. (2013) 190:291-301. doi: 10.1016/j.juro.2013.01.098

32. Bardin A, Moll F, Margueron R, Delfour C, Chu ML, Maudelonde T, et al. Transcriptional and posttranscriptional regulation of fibulin-1 by estrogens leads to differential induction of messenger ribonucleic acid variants in ovarian and breast cancer cells. Endocrinology. (2005) 146:760-8. doi: 10.1210/en.2004-1239

33. Greene LM, Twal WO, Duffy MJ, McDermott EW, Hill AD, O’Higgins NJ, et al. Elevated expression and altered processing of fibulin-1 protein in human breast cancer. Br J Cancer. (2003) 88:871-8. doi: 10.1038/sj.bjc.6600802

34. Muriel JM, Dong C, Hutter H, Vogel BE. Fibulin-1C and Fibulin-1D splice variants have distinct functions and assemble in a hemicentin-dependent manner. Development. (2005) 132:4223-34. doi: 10.1242/dev.02007

35. Mohamedi Y, Fontanil T, Cobo T, Vega JA, Cobo JL, García-Suárez O, et al. The molecular interaction of ADAMTS-1 and fibulin-1 and its potential contribution to breast cancer biology. J Cancer Metastasis Treat. (2019) 5:37. doi: 10.20517/2394-4722.2018.81

36. Lee NV, Rodriguez-Manzaneque JC, Thai SN, Twal WO, Luque A, Lyons KM, et al. Fibulin- 1 acts as a cofactor for the matrix metalloprotease ADAMTS-1. $J$ Biol Chem. (2005) 280:34796-804. doi: 10.1074/jbc.M506980200

37. Rodriguez-Manzaneque JC, Milchanowski AB, Dufour EK, Leduc R, Iruela-Arispe ML. Characterization of METH-1/ADAMTS1 processing reveals two distinct active forms. J Biol Chem. (2000) 275:33471-9. doi: 10.1074/jbc.M002599200

38. Liu YJ, Xu Y, Yu Q. Full-length ADAMTS-1 and the ADAMTS-1 fragments display pro- and antimetastatic activity, respectively. Oncogene. (2006) 25:2452-67. doi: 10.1038/sj.onc. 1209287

39. Vazquez F, Hastings G, Ortega MA, Lane TF, Oikemus S, Lombardo M, et al. METH-1, a human ortholog of ADAMTS-1, and METH-2 are members of a new family of proteins with angio-inhibitory activity. J Biol Chem. (1999) 274:23349-57.

40. Luque A, Carpizo DR, Iruela-Arispe ML. ADAMTS1/METH1 inhibits endothelial cell proliferation by direct binding and sequestration of VEGF165. J Biol Chem. (2003) 278:23656-65. doi: 10.1074/jbc.M212964200

41. Freitas VM, do Amaral JB, Silva TA, Santos ES, Mangone FR, de Pinheiro J, et al. Decreased expression of ADAMTS-1 in human breast tumors stimulates migration and invasion. Mol Cancer. (2013) 12:2. doi: 10.1186/1476-4598-12-2

42. Silva SV, Lima MA, Cella N, Jaeger RG, Freitas VM. ADAMTS-1 is found in the nuclei of normal and tumoral breast cells. PLoS ONE. (2016) 11:e0165061. doi: 10.1371/journal.pone.0165061

43. Lima MA, Dos Santos L, Turri JA, Nonogaki S, Buim M, Lima JF, et al. Prognostic value of ADAMTS proteases and their substrates in epithelial ovarian cancer. Pathobiology. (2016) 83:316-26. doi: 10.1159/000446244

44. Fu Y, Nagy JA, Brown LF, Shih SC, Johnson PY, Chan CK, et al. Proteolytic cleavage of versican and involvement of ADAMTS-1 in VEGF-A/VPFinduced pathological angiogenesis. J Histochem Cytochem. (2011) 59:463-73. doi: $10.1369 / 0022155411401748$

45. Rocks N, Paulissen G, Quesada-Calvo F, Munaut C, Gonzalez ML, Gueders M, et al. ADAMTS-1 metalloproteinase promotes tumor development through the induction of a stromal reaction in vivo. Cancer Res. (2008) 68:9541-50. doi: 10.1158/0008-5472.CAN-08-0548

46. Binder MJ, McCoombe S, Williams ED, McCulloch DR, Ward AC. The extracellular matrix in cancer progression: role of hyalectan proteoglycans and ADAMTS enzymes. Cancer Lett. (2017) 385:55-64. doi: 10.1016/j.canlet.2016.11.001 
47. Nandadasa S, Foulcer S, Apte SS. The multiple, complex roles of versican and its proteolytic turnover by ADAMTS proteases during embryogenesis. Matrix Biol. (2014) 35:34-41. doi: 10.1016/j.matbio.2014.01.005

48. McCulloch DR, Nelson CM, Dixon LJ, Silver DL, Wylie JD, Lindner $\mathrm{V}$, et al. ADAMTS metalloproteases generate active versican fragments that regulate interdigital web regression. Dev. Cell. (2009) 17:687-98. doi: 10.1016/j.devcel.2009.09.008

49. Hope C, Foulcer S, Jagodinsky J, Chen SX, Jensen JL, Patel $\mathrm{S}$, et al. Immunoregulatory roles of versican proteolysis in the myeloma microenvironment. Blood. (2016) 128:680-5. doi: 10.1182/blood-2016-03-705780

50. Hope C, Emmerich PB, Papadas A, Pagenkopf A, Matkowskyj KA, Van De Hey DR, et al. Versican-derived matrikines regulate Batf3-dendritic cell differentiation and promote T Cell infiltration in colorectal cancer. J Immunol. (2017) 199:1933-41. doi: 10.4049/jimmunol.1700529

51. Wu YJ, La Pierre DP, Wu J, Yee AJ, Yang BB. The interaction of versican with its binding partners. Cell Res. (2005) 15:483-94. doi: 10.1038/sj.cr.7290318

52. Longpre JM, McCulloch DR, Koo BH, Alexander JP, Apte SS, Leduc R. Characterization of proADAMTS5 processing by proprotein convertases. Int J Biochem Cell Biol. (2009) 41:1116-26. doi: 10.1016/j.biocel.2008. 10.008

53. Ducros E, Berthaut A, Mirshahi P, Lemarchand S, Soria J, Legeais $\mathrm{JM}$, et al. Expression of extracellular matrix proteins fibulin-1 and fibulin-2 by human corneal fibroblasts. Curr Eye Res. (2007) 32:481-90. doi: 10.1080/02713680701411269

54. Kern CB, Twal WO, Mjaatvedt CH, Fairey SE, Toole BP, Iruela-Arispe ML, et al. Proteolytic cleavage of versican during cardiac cushion morphogenesis. Dev Dyn. (2006) 235:2238-47. doi: 10.1002/dvdy.20838

55. Zhang HY, Kluge M, Timpl R, Chu ML, Ekblom P. The extracellular matrix glycoproteins BM-90 and tenascin are expressed in the mesenchyme at sites of endothelial-mesenchymal conversion in the embryonic mouse heart. Differentiation. (1993) 52:211-20.

56. Cooley MA, Fresco VM, Dorlon ME, Twal WO, Lee NV, Barth JL, et al. Fibulin-1 is required during cardiac ventricular morphogenesis for versican cleavage, suppression of ErbB2 and Erk1/2 activation, and to attenuate trabecular cardiomyocyte proliferation. Dev Dyn. (2012) 241:30314. doi: $10.1002 /$ dvdy. 23716

57. Thai SN, Iruela-Arispe ML. Expression of ADAMTS1 during murine development. Mech Dev. (2002) 115:181-5. doi: 10.1016/S0925-4773(02)00115-6

58. Spence SG, Argraves WS, Walters L, Hungerford JE, Little CD. Fibulin is localized at sites of epithelial-mesenchymal transitions in the early avian embryo. Dev Biol. (1992) 151:473-84.

59. Bouchey D, Argraves WS, Little CD. Fibulin-1, vitronectin, and fibronectin expression during avian cardiac valve and septa development. Anat Rec. (1996) 244:540-51.

60. Pan TC, Sasaki T, Zhang RZ, Fassler R, Timpl R, Chu ML. Structure and expression of fibulin-2, a novel extracellular matrix protein with multiple EGF-like repeats and consensus motifs for calcium binding. J Cell Biol. (1993) 123:1269-77.

61. Olin AI, Morgelin M, Sasaki T, Timpl R, Heinegard D, Aspberg A. The proteoglycans aggrecan and Versican form networks with fibulin-2 through their lectin domain binding. J Biol Chem. (2001) 276:1253-61. doi: 10.1074/jbc.M006783200

62. Sasaki T, Gohring W, Pan TC, Chu ML, Timpl R. Binding of mouse and human fibulin-2 to extracellular matrix ligands. J Mol Biol. (1995) 254:892-9. doi: 10.1006/jmbi.1995.0664

63. Hopf M, Gohring W, Kohfeldt E, Yamada Y, Timpl R. Recombinant domain IV of perlecan binds to nidogens, laminin-nidogen complex, fibronectin, fibulin-2 and heparin. Eur J Biochem. (1999) 259:917-25.

64. Pfaff M, Sasaki T, Tangemann K, Chu ML, Timpl R. Integrin-binding and celladhesion studies of fibulins reveal a particular affinity for alpha IIb beta 3. Exp Cell Res. (1995) 219:87-92. doi: 10.1006/excr.1995.1208

65. Gallagher WM, Currid CA, Whelan LC. Fibulins and cancer: friend or foe? Trends Mol Med. (2005) 11:336-40. doi: 10.1016/j.molmed.2005.06.001

66. Senapati S, Gnanapragassam VS, Moniaux N, Momi N, Batra SK. Role of MUC4-NIDO domain in the MUC4-mediated metastasis of pancreatic cancer cells. Oncogene. (2012) 31:3346-56. doi: 10.1038/onc.2011.505
67. Law EW, Cheung AK, Kashuba VI, Pavlova TV, Zabarovsky ER, Lung $\mathrm{HL}$, et al. Anti-angiogenic and tumor-suppressive roles of candidate tumorsuppressor gene, Fibulin-2, in nasopharyngeal carcinoma. Oncogene. (2012) 31:728-38. doi: 10.1038/onc.2011.272

68. Yi CH, Smith DJ, West WW, Hollingsworth MA. Loss of fibulin-2 expression is associated with breast cancer progression. Am J Pathol. (2007) 170:1535-45. doi: 10.2353/ajpath.2007.060478

69. Dunwell TL, Hesson LB, Pavlova T, Zabarovska V, Kashuba V, Catchpoole $\mathrm{D}$, et al. Epigenetic analysis of childhood acute lymphoblastic leukemia. Epigenetics. (2009) 4:185-93. doi: 10.4161/epi.4.3.8752

70. Tan H, Zhang J, Fu D, Zhu Y. Loss of fibulin-2 expression is involved in the inhibition of breast cancer invasion and forms a new barrier in addition to the basement membrane. Oncol Lett. (2017) 14:2663-8. doi: 10.3892/ol.2017.6539

71. Ren T, Lin S, Wang Z, Shang A. Differential proteomics analysis of lowand high-grade of astrocytoma using iTRAQ quantification. Oncotargets Ther. (2016) 9:5883-95. doi: 10.2147/OTT.S111103

72. Fontanil T, Rua S, Llamazares M, Moncada-Pazos A, Quiros PM, GarciaSuarez O, et al. Interaction between the ADAMTS-12 metalloprotease and fibulin-2 induces tumor-suppressive effects in breast cancer cells. Oncotarget. (2014) 5:1253-64. doi: 10.18632/oncotarget.1690

73. Noel A, Gutierrez-Fernandez A, Sounni NE, Behrendt N, Maquoi E, Lund IK, et al. New and paradoxical roles of matrix metalloproteinases in the tumor microenvironment. Front Pharmacol. (2012) 3:140. doi: 10.3389/fphar.2012.00140

74. Luan Y, Kong L, Howell DR, Ilalov K, Fajardo M, Bai XH, et al. Inhibition of ADAMTS-7 and ADAMTS-12 degradation of cartilage oligomeric matrix protein by alpha-2-macroglobulin. Osteoarthr Cartilage. (2008) 16:1413-20. doi: 10.1016/j.joca.2008.03.017

75. Llamazares M, Obaya AJ, Moncada-Pazos A, Heljasvaara R, Espada J, LopezOtin C, et al. The ADAMTS12 metalloproteinase exhibits anti-tumorigenic properties through modulation of the Ras-dependent ERK signalling pathway. J Cell Sci. (2007) 120(Pt 20):3544-52. doi: 10.1242/jcs.005751

76. Moncada-Pazos A, Obaya AJ, Fraga MF, Viloria CG, Capella G, Gausachs M, et al. The ADAMTS12 metalloprotease gene is epigenetically silenced in tumor cells and transcriptionally activated in the stroma during progression of colon cancer. J Cell Sci. (2009) 122(Pt 16):2906-13. doi: 10.1242/jcs.050468

77. O’Brien LE, Tang K, Kats ES, Schutz-Geschwender A, Lipschutz JH, Mostov KE. ERK and MMPs sequentially regulate distinct stages of epithelial tubule development. Dev Cell. (2004) 7:21-32. doi: 10.1016/j.devcel.2004.06.001

78. El Hour M, Moncada-Pazos A, Blacher S, Masset A, Cal S, Berndt S, et al. Higher sensitivity of Adamts12-deficient mice to tumor growth and angiogenesis. Oncogene. (2010) 29:3025-32. doi: 10.1038/onc.2010.49

79. Fontanil T, Alvarez-Teijeiro S, Villaronga MA, Mohamedi Y, Solares L, Moncada-Pazos A, et al. Cleavage of Fibulin-2 by the aggrecanases ADAMTS4 and ADAMTS- 5 contributes to the tumorigenic potential of breast cancer cells. Oncotarget. (2017) 8:13716-29. doi: 10.18632/oncotarget.14627

80. Tortorella MD, Burn TC, Pratta MA, Abbaszade I, Hollis JM, Liu R, et al. Purification and cloning of aggrecanase-1: a member of the ADAMTS family of proteins. Science. (1999) 284:1664-6.

81. Bondeson J, Wainwright S, Hughes C, Caterson B. The regulation of the ADAMTS4 and ADAMTS5 aggrecanases in osteoarthritis: a review. Clin Exp Rheumatol. (2008) 26:139-45.

82. Held-Feindt J, Paredes EB, Blomer U, Seidenbecher C, Stark AM, Mehdorn HM, et al. Matrix-degrading proteases ADAMTS4 and ADAMTS5 (disintegrins and metalloproteinases with thrombospondin motifs 4 and 5) are expressed in human glioblastomas. Int J Cancer. (2006) 118:55-61. doi: $10.1002 /$ ijc. 21258

83. Nakada M, Miyamori H, Kita D, Takahashi T, Yamashita J, Sato $\mathrm{H}$, et al. Human glioblastomas overexpress ADAMTS-5 that degrades brevican. Acta Neuropathol. (2005) 110:239-46. doi: 10.1007/s00401-0051032-6

84. Sasaki T, Mann K, Murphy G, Chu ML, Timpl R. Different susceptibilities of fibulin-1 and fibulin-2 to cleavage by matrix metalloproteinases and other tissue proteases. Eur J Biochem. (1996) 240:427-34.

85. Lecka-Czernik B, Lumpkin CK Jr, Goldstein S. An overexpressed gene transcript in senescent and quiescent human fibroblasts encoding a novel protein in the epidermal growth factor-like repeat family stimulates DNA synthesis. Mol Cell Biol. (1995) 15:120-8. 
86. Giltay R, Timpl R, Kostka G. Sequence, recombinant expression and tissue localization of two novel extracellular matrix proteins, fibulin-3 and fibulin-4. Matrix Biol. (1999) 18:469-80.

87. Albig AR, Neil JR, Schiemann WP. Fibulins 3 and 5 antagonize tumor angiogenesis in vivo. Cancer Res. (2006) 66:2621-9. doi: 10.1158/0008-5472.CAN-04-4096

88. Hu B, Thirtamara-Rajamani KK, Sim H, Viapiano MS. Fibulin3 is uniquely upregulated in malignant gliomas and promotes tumor cell motility and invasion. Mol Cancer Res. (2009) 7:1756-70. doi: 10.1158/1541-7786.MCR-09-0207

89. Seeliger H, Camaj P, Ischenko I, Kleespies A, De Toni EN, Thieme $\mathrm{SE}$, et al. EFEMP1 expression promotes in vivo tumor growth in human pancreatic adenocarcinoma. Mol Cancer Res. (2009) 7:189-98. doi: 10.1158/1541-7786.MCR-08-0132

90. En-lin S, Sheng-guo C, Hua-qiao W. The expression of EFEMP1 in cervical carcinoma and its relationship with prognosis. Gynecol Oncol. (2010) 117:417-22. doi: 10.1016/j.ygyno.2009. 12.016

91. Li J, Qi C, Liu X, Li C, Chen J, Shi M. Fibulin-3 knockdown inhibits cervical cancer cell growth and metastasis in vitro and in vivo. Sci Rep. (2018) 8:10594. doi: 10.1038/s41598-018-28906-9

92. Wang S, Zhang D, Han S, Gao P, Liu C, Li J, et al. Fibulin-3 promotes osteosarcoma invasion and metastasis by inducing epithelial to mesenchymal transition and activating the Wnt/beta-catenin signaling pathway. Sci Rep. (2017) 7:6215. doi: 10.1038/s41598-017-06353-2

93. Arechederra M, Priego N, Vazquez-Carballo A, Sequera C, Gutierrez-Uzquiza A, Cerezo-Guisado MI, et al. p38 MAPK down-regulates fibulin 3 expression through methylation of gene regulatory sequences: role in migration and invasion. J Biol Chem. (2015) 290:4383-97. doi: 10.1074/jbc.M114.582239

94. Tian H, Liu J, Chen J, Gatza ML, Blobe GC. Fibulin-3 is a novel TGF-beta pathway inhibitor in the breast cancer microenvironment. Oncogene. (2015) 34:5635-47. doi: 10.1038/onc.2015.13

95. Chen X, Meng J, Yue W, Yu J, Yang J, Yao Z, et al. Fibulin-3 suppresses Wnt/beta-catenin signaling and lung cancer invasion. Carcinogenesis. (2014) 35:1707-16. doi: 10.1093/carcin/bgu023

96. Kostka G, Giltay R, Bloch W, Addicks K, Timpl R, Fassler R, et al. Perinatal lethality and endothelial cell abnormalities in several vessel compartments of fibulin-1-deficient mice. Mol Cell Biol. (2001) 21:7025-34. doi: 10.1128/MCB.21.20.7025-7034.2001

97. Sicot FX, Tsuda T, Markova D, Klement JF, Arita M, Zhang RZ, et al. Fibulin2 is dispensable for mouse development and elastic fiber formation. Mol Cell Biol. (2008) 28:1061-7. doi: 10.1128/MCB.01876-07

Conflict of Interest Statement: The authors declare that the research was conducted in the absence of any commercial or financial relationships that could be construed as a potential conflict of interest.

Copyright (C) 2019 Fontanil, Mohamedi, Cobo, Cal and Obaya. This is an open-access article distributed under the terms of the Creative Commons Attribution License (CC BY). The use, distribution or reproduction in other forums is permitted, provided the original author(s) and the copyright owner(s) are credited and that the original publication in this journal is cited, in accordance with accepted academic practice. No use, distribution or reproduction is permitted which does not comply with these terms. 